\title{
Malignant extra-gastrointestinal stromal tumor of the liver: A case report
}

\author{
YINGCHAO WANG* ${ }^{*}$ YAHUI LIU*, YANPING ZHONG and BAI JI \\ Department of Hepatobiliary and Pancreatic Surgery, The First Hospital of Jilin University, \\ Changchun, Jilin 130021, P.R. China
}

Received January 20, 2015; Accepted February 24, 2016

DOI: $10.3892 / \mathrm{ol} .2016 .4531$

\begin{abstract}
Extra-gastrointestinal stromal tumors (EGISTs) predominantly occur outside of the gastrointestinal tract, and their biological and histological characteristics are similar to those of GISTs. Primary EGIST occurrence in the liver is extremely rare. The present study reports a case of primary EGIST in the caudate lobe of the liver in a 61-year-old Chinese man. Contrast-enhanced computed tomography revealed a $7.3 \times 5.1-\mathrm{cm}$ heterogeneously enhanced neoplasm with solid and cystic components located in the caudate lobe of the liver. The patient underwent caudate lobe (specifically, Spiegel lobe) resection. Immunohistochemical analysis of the resected tumor revealed a strong positivity for cluster of differentiation (CD)117, discovered on GIST-1 and CD34. Thus, based on the histological and immunohistochemical findings, the final diagnosis was primary hepatic EGIST. Follow up was conducted at 3-month intervals for the first year and 6-months thereafter. The patient was asymptomatic without any sign of recurrence during the follow-up period. Lab tests were in normal range, and no mass was found in CT scan.
\end{abstract}

\section{Introduction}

Gastrointestinal stromal tumors (GISTs) are the most common gastrointestinal tract tumors of mesenchymal origin. Those occurring outside of the gastrointestinal tract comprise a specific type of GIST, known as extra-gastrointestinal stromal tumors (EGISTs) (1). EGISTs share similar biological and histological characteristics with GISTs, and predominantly occur in the omentum, retroperitoneum or mesentery (2-5), and less often in the abdominal wall (6), pleura (7), pancreas (8), liver (9), adrenal glands (10), bladder (11) and prostate (12).

Correspondence to: Dr Bai Ji, Department of Hepatobiliary and Pancreatic Surgery, The First Hospital of Jilin University, 71 Xinmin Street, Changchun, Jilin 130021, P.R. China

E-mail: jirulin@yeah.net

*Contributed equally

Key words: extra-gastrointestinal stromal tumor, malignant, liver
The incidence of EGISTs is low, accounting of $~ 5-7 \%$ of all GISTs (13). GIST metastasis to the liver is considered common, whereas primary EGIST of the liver is considerably rare. According to a study by Li et al (14), only 6 cases worldwide of primary hepatic EGIST were reported between 2003 and 2012. The present study reports a case of primary EGIST located in the caudate lobe of the liver in a 61-year-old Chinese man.

\section{Case report}

A 61-year-old Chinese man was admitted to The First Hospital of Jilin University (Changchun, China) on February 11, 2014 , for the treatment of an abdominal mass detected by abdominal ultrasonography during a health examination. The abdominal ultrasound identified a $3 \times 7-\mathrm{cm}$ mass in the caudate lobe of the liver. Following admission, a contrast-enhanced computed tomography (CECT) scan revealed a $7.3 \times 5.1-\mathrm{cm}$ mass with solid and cystic components in the caudate lobe of the liver. The mass displayed post-contrast heterogeneous enhancement (Fig. 1), therefore, the mass was considered to be malignant. The possibility of a neurogenic tumor was also considered. The findings of physical and laboratory tests for hepatitis $\mathrm{B}$ and $\mathrm{C}$ virus, liver function and tumor markers, such as $\alpha$-fetoprotein and carcinoembryonic antigen, were all within the normal limits. Therefore, the patient underwent an exploratory laparotomy on February 17, 2014. Intraoperatively, the mass was hard with an uneven surface, measured $7 \times 5 \mathrm{~cm}$ and was located in the caudate lobe of the liver (Fig. 2). No abnormal lesions were identified in the stomach, duodenum, small intestine, colon, pancreas, peritoneum, omentum or any other organs in the abdominal cavity. The patient underwent caudate lobe resection, in particular Spiegel lobe resection. Sectioning of the resected tumor revealed that inside of the mass was divided by numerous fibers and filled with necrotic tissue (Fig. 2). Immunohistochemistry of the resected tissue showed a strong positivity for cluster of differentiation (CD)117, discovered on GIST-1 (DOG1) and CD34. The Ki-67 labeling index was found to be $2 \%$. Hematoxylin and eosin staining demonstrated the tumor was composed of spindle cells (Fig. 3). These histological and immunohistochemical features led to a final diagnosis of high-grade primary hepatic EGIST. The present study was approved by the ethics committee of the first hospital of Jilin University, Changchun, China. 


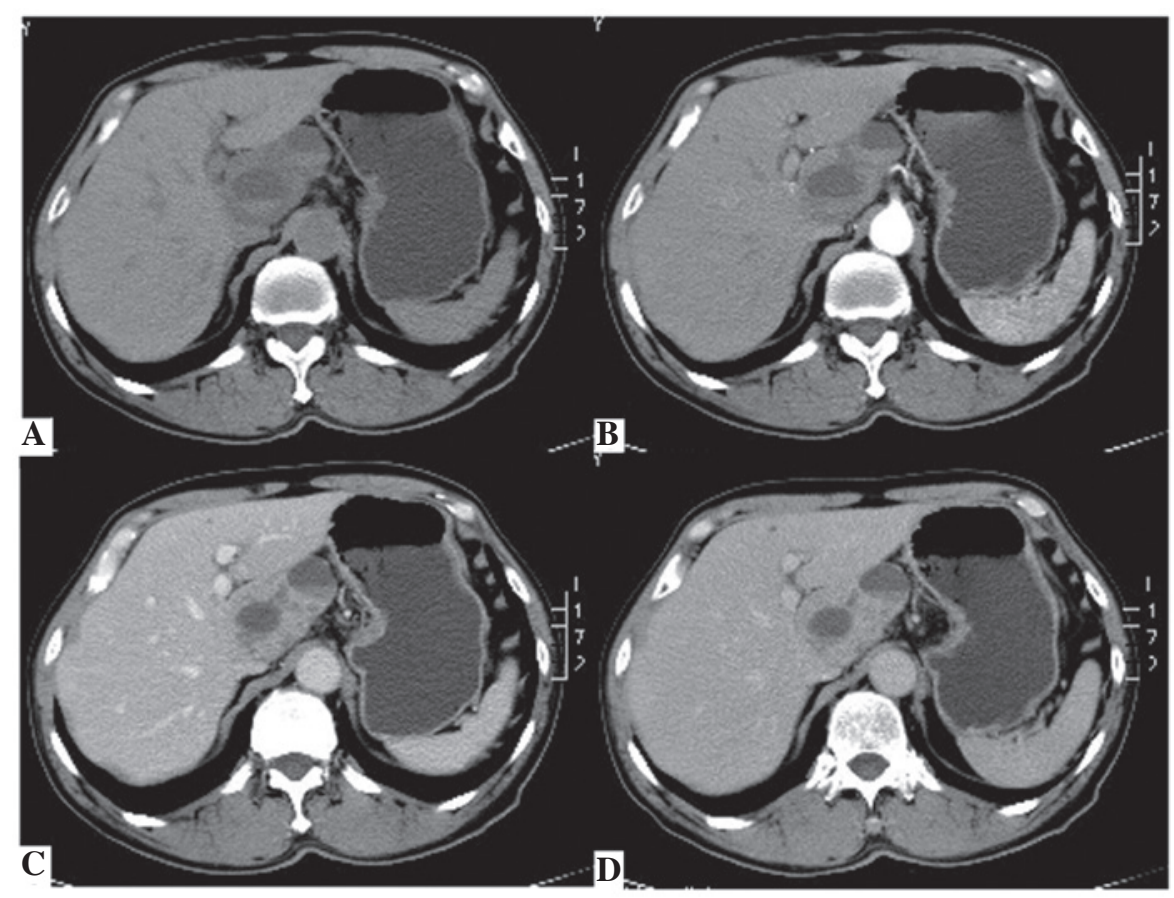

Figure 1. Contrast-enhanced computed tomography revealing (A) a 7.3x5.1-cm mass with solid and cystic components in the caudate lobe of the liver, with (B) an uneven enhancement in the arterial phase of scanning, which was progressively strengthened in the (C) venous and (D) delayed phases.

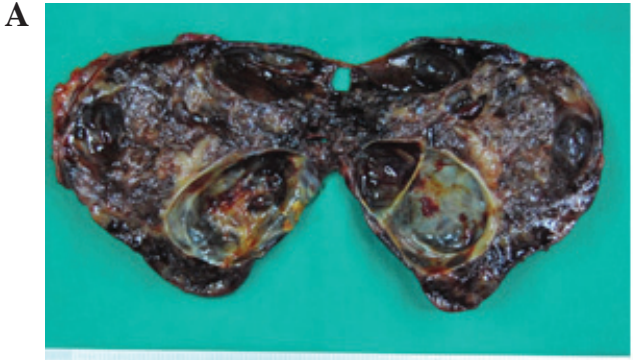

$10 \quad 11 \quad 12 \quad 13 \quad 14 \quad 15 \quad 16 \quad 17 \quad 18 \quad 192021 \quad 22 \quad 23 \quad 24 \quad 25$
B

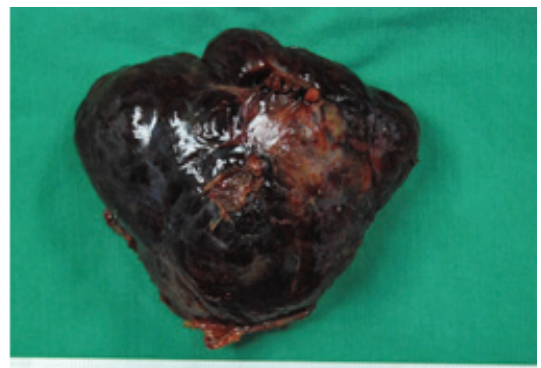

$10 \quad 11 \quad 12 \quad 13 \quad 14 \quad 1516 \quad 17 \quad 181920 \quad 2122$

Figure 2. Images of the tumor following resection. (A) A hard mass measuring $7 \times 5 \mathrm{~cm}$ was found to be located in the caudate lobe of the liver. (B) Internally, the tumor was divided by fibers and filled with necrotic tissue.

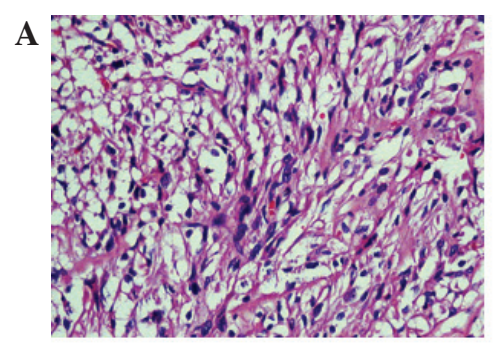

B

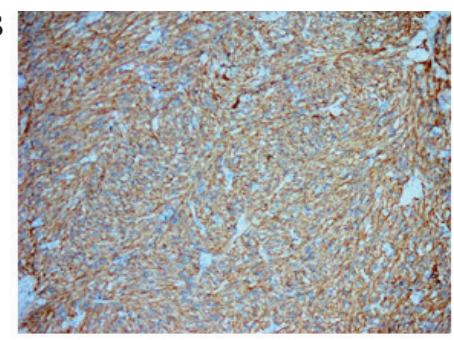

C

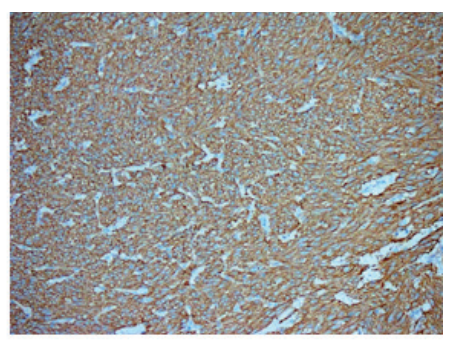

D

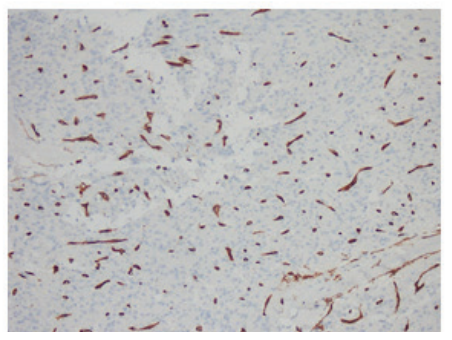

$\mathbf{E}$

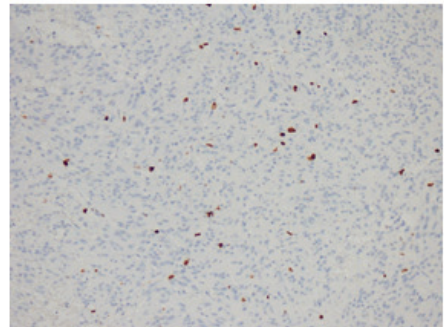

Figure 3. Histological and immunohistochemical findings for the hepatic extra-gastrointestinal stromal tumor. (A) Hematoxylin and eosin staining (magnification, x400). (B) Cluster of differentiation (CD)117-, (C) discovered on GIST-1-, (D) CD34- and (E) Ki67-positive tumor cells (magnification, x200). 
The patient was discharged from the hospital in the absence of any clinically significant events, and follow up was at 3-month intervals during the first year and at 6-month intervals thereafter. Follow-up tests included routine CBC, liver function test as well as abdominal CT scan. The patient was asymptomatic without any sign of recurrence during the follow-up period. Lab tests were in normal range, and no mass was found in CT scan.

\section{Discussion}

EGISTs predominantly occur in male adults aged $>50$ years $(15,16)$ and the tumor size is typically $>5 \mathrm{~cm}$. The phenotypic characteristics of GISTs include elevated expression of CD117, a receptor tyrosine kinase protein that is encoded by the c-Kit gene in humans. Generally, EGISTs exhibit a c-Kit expression similar to that of GISTs. Gain-of-function mutations in the c-Kit and platelet-derived growth factor receptor- $\alpha$ (PDGFR- $\alpha$ ) genes have been previously reported in GISTs, and are considered to constitute an important mechanism for their development (17). Yamamoto et al (18) identified that the c-Kit and PDGFR- $\alpha$ mutation pattern in EGISTs was similar to that in GISTs, indicating the potential role of c-Kit and PDGFR- $\alpha$ mutations in the tumorigenesis of EGISTs as well.

Several hypotheses have been proposed regarding the tumorigenesis of EGISTs. A previous study has suggested that GISTs may originate from interstitial cells of Cajal (ICCs), due to the striking morphological and immunophenotypic similarities between GISTs and ICCs (19). From a molecular genetic perspective, EGISTs comprise a specific type of GIST, therefore, theoretically, they could be share the same mesenchymal origin; however, ICCs form a network surrounding the gastrointestinal plexus, which cannot explain the occurrence of EGISTs. Agaimy et al (20) proposed that EGISTs are extremely rare, and that various reported EGISTs may have actually been GISTs that arose from the outermost muscle layer of the gastrointestinal tract but were detached from the original site during their growth. For example, mesenteric and omental EGISTs may derive from GISTs of the small intestine and stomach, respectively. An alternative explanation is that EGISTs originate from multipotent mesenchymal stem cells located outside of the gastrointestinal tract.

Thus far, no common symptoms specific to EGISTs have been identified. The majority of cases are initially detected by the presence of a mass upon physical examination. In the current case, the patient was found to have a hepatic lesion during a health examination. CECT further confirmed a $7.3 \times 5.1-\mathrm{cm}$ heterogeneously enhanced mass with solid and cystic components in the caudate lobe of the liver. The mass was filled with necrotic tissue and fed by numerous blood vessels. The lesion exhibited an uneven enhancement in the arterial phase of scanning, presenting as a large, well-circumscribed low-density mass with a partial high-density area. In the venous and delayed phases, enhancement was progressively strengthened (Fig. 1).

In the majority of cases of EGISTs, preoperative diagnosis is not possible, therefore, patients may be easily misdiagnosed with different types of cancer, such as lymphoma, neurogenic tumors and malignant fibrous histiocytoma. In the present case, the mass was diagnosed as a malignant lesion of the liver prior to surgical intervention, however, the nature of the tumor could not be determined preoperatively. Occasionally, preoperative ultrasound-guided fine needle aspiration cytology can assist the diagnosis of EGISTs. The final diagnosis, however, is generally based on histological, immunohistochemical and molecular findings. In 95\% of EGIST cases, the CD117 protein exhibits a strong, diffuse positive immunohistochemical reaction, and $60-70 \%$ of EGISTs are CD34-positive (21). In the present case, immunohistochemical examination of the tumor tissue revealed strong positivity for CD117, DOG1 and CD34. The final diagnosis of primary hepatic EGIST was based on the fact that no abnormal lesions were identified in any other organs in the abdominal cavity except the liver.

The standard treatment for both GISTs and primary non-metastatic EGISTs is complete surgical resection with a microscopic negative margin, which was the case for the patient in the present report. During the surgical resection of the tumor, clinicians should be careful not to cause implantation metastasis. In addition, large tumors should not be removed via laparoscopic resection (22). Imatinib, a tyrosine kinase inhibitor of c-Kit, has also been used for the treatment of GISTs and EGISTs. Imatinib can improve overall recurrence-free survival, even in patients with advanced GISTs $(23,24)$; however, certain studies have indicated that adjuvant therapy with tyrosine kinase inhibitors appears to be unnecessary in cases when a negative microscopic margin ( $\mathrm{R} 0$ resection) is achieved (24). EGISTs are commonly accompanied by certain adverse prognostic factors, such as large primary tumors and distant or lymph node metastases. As a result, EGISTs have a worse prognosis than GISTs (7).

In conclusion, primary hepatic EGISTs are extremely rare. Histological and immunohistochemical features, including positive staining for CD117 and CD34, can aid in obtaining an accurate diagnosis. Complete tumor resection with a microscopic negative margin is considered the optimal treatment option.

\section{Acknowledgements}

The authors would like to thank the staff of the Pathological Department of The First Hospital of Jilin University (Changchun, China) for their the assistance.

\section{References}

1. Emory TS, Sobin LH, Lukes L, Lee DH, O'Leary TJ. Prognosis of gastrointestinal smooth-muscle (stromal) tumors: Dependence on anatomic site. Am J Surg Pathol 23: 82-7, 1999.

2. Fukuda H, Suwa T, Kimura F, Sugiura T, Shinoda T and Kaneko K: Gastrointestinal stromal tumor of the lesser omentum: Report of a case. Surg Today 31: 715-718, 2001

3. Nakaya I, Iwata Y, Abe T, Yokoyama H, Oda Y and Nomura G: Malignant gastrointestinal stromal tumor originating in the lesser omentum, complicated by rapidly progressive glomerulonephritis and gastric carcinoma. Intern Med 43: 102-105, 2004.

4. Takao H, Yamahira K, Doi I and Watanabe T: Gastrointestinal stromal tumor of the retroperitoneum: CT and MR findings. Eur Radiol 14: 1926-1929, 2004.

5. Llenas-Garcia J, Guerra-Vales JM, Moreno A, Ibarrola C, Castelbon FJ, Fernández-Ruiz M, Meneu JC, Ballestin C and Moreno E: Primary extragastrointestinal stromal tumors in the omentum and mesentery: A clinicopathological and immunohistochemical study. Hepatogastroenterology 55: 1002-1005, 2008. 
6. Kumar AS, Padmini R, Veena G and Murugesan N: Extragastrointestinal stromal tumour of the abdominal wall-a case report J Clin Diagn Res 7: 2970-2972, 2013.

7. Yi JH, Sim J, Park BB, Lee YY, Jung WS, Jang HJ, Ha TK and Paik SS: The primary extra-gastrointestinal stromal tumor of pleura: A case report and a literature review. Jpn J Clin Oncol 43: 1269-1272, 2013.

8. Yamaura K, Kato K, Miyazawa M, Haba Y, et al: Stromal tumor of the pancreas with expression of c-kit protein: Report of a case. J Gastroenterol Hepatol 19: 467-470, 2004.

9. Chen J, Du YJ, Song JT, E LN and Liu BR: Primary malignant liver mesenchymal tumor: A case report. World J Gastroenterol 16: 5263-5266, 2010.

10. Sereg M, Buzogány I, Gonda G, Sápi Z, Csöregh E, Jakab Z, Rácz K and Tóth M: Gastrointestinal stromal tumor presenting as a hormonally inactive adrenal mass. Endocrine 39: 1-5, 2011.

11. Mekni A, Chelly I, Azzouz H, Ben Ghorbel I, Bellil S, Haouet S Kchir N, Zitouna M and Bellil K: Extragastrointestinal stromal tumor of the urinary wall bladder: Case report and review of the literature. Pathologica 100: 173-175, 2008.

12. Zhou J and Teng X: Primary extragastrointestinal stromal tumor of the prostate: A case report. Anal Quant Cytopathol Histpathol 36: 55-60, 2014.

13. Emory TS, Sobin LH, Lukes L, Lee DH and O'Leary TJ: Prognosis of gastrointestinal smooth-muscle (stromal) tumors: Dependence on anatomic site. Am J Surg Pathol 23: 82-87, 1999.

14. Li ZY, Liang QL, Chen GQ, Zhou Y and Liu QL: Extra-gastrointestinal stromal tumor of the liver diagnosed by ultrasound-guided fine needle aspiration cytology: A case report and review of the literature. Arch Med Sci 8: 392-397, 2012.

15. Fagkrezos D, Touloumis Z, Giannila M, Penlidis C, et al: Extra-gastrointestinal stromal tumor of the omentum: A rare case report and review of the literature. Rare Tumors 4: e44, 2012.

16. Goh BK, Chow PK, et al: A single-institution experience with eight CD117-positive primary extragastrointestinal stromal tumors: Critical appraisal and a comparison with their gastrointestinal counterparts. J Gastrointest Surg 13: 1094-1098, 2009.
17. Isozaki K and Hirota S: Gain-of-Function mutations of receptor tyrosine kinases in gastrointestinal stromal tumors. Curr Genomics 7: 469-475, 2006.

18. Yamamoto H, Oda Y, Kawaguchi K, Nakamura N, Takahira T, Tamiya S, Saito T, Oshiro Y, Ohta M, Yao T and Tsuneyoshi M: c-kit and PDGFRA mutations in extragastrointestinal stromal tumor (gastrointestinal stromal tumor of the soft tissue). Am J Surg Pathol 28: 479-488, 2004.

19. Kindblom LG, Remotti HE, Aldenborg F and Meis-Kindblom JM: Gastrointestinal pacemaker cell tumor (GIPACT): Gastrointestinal stromal tumors show phenotypic characteristics of the interstitial cells of Cajal. Am J Pathol 152: 1259-1269, 1998.

20. Agaimy A and Wünsch PH: Gastrointestinal stromal tumours: A regular origin in the muscularis propria, but an extremely diverse gross presentation. A review of 200 cases to critically re-evaluate the concept of so-called extra-gastrointestinal stromal tumours. Langenbecks Arch Surg 391: 322-329, 2006.

21. Tian YT, Liu H, Shi SS, Xie YB, Xu Q, Zhang JW, Zhao DB, Wang CF and Chen YT: Malignant extra-gastrointestinal stromal tumor of the pancreas: Report of two cases and review of the literature. World J Gastroenterol 20: 863-868, 2014.

22. Casali PG and Blay JY; ESMO/CONTICANET/EUROBONET Consensus Panel of Experts: Gastrointestinal stromal tumours: ESMO clinical practice guidelines for diagnosis, treatment and follow-up. Ann Oncol 21 (Suppl 5): v98-v102, 2010.

23. Blanke CD, Demetri GD, von Mehren M, Heinrich MC, Eisenberg B, Fletcher JA, Corless CL, Fletcher CD, Roberts PJ, Heinz D, et al: Long-term results from a randomized phase II trial of standard- versus higher-dose imatinib mesylate for patients with unresectable or metastatic gastrointestinal stromal tumors expressing KIT. J Clin Oncol 26: 620-625, 2008.

24. Li J, Gong JF, Wu AW and Shen L: Post-operative imatinib in patients with intermediate or high risk gastrointestinal stromal tumor. Eur J Surg Oncol 37: 319-324, 2011. 